\title{
Information: Concept Clarification and Theoretical Representation
}

\author{
Mark Burgin
}

\author{
mburgin@math.ucla.edu, Department of Mathematics, University of California, Los Angeles
}

\begin{abstract}
The general theory of information is a synthetic approach, which organizes and encompasses all main directions in information theory. It is developed on three levels: conceptual, methodological and theoretical. On the conceptual level, the concept of information is purified and information operations are separated and described. On the methodological level, it is formulated as system of principles, explaining what information is and how to measure information. On the theoretical level, mathematical models of information are constructed and studied. The goal of this paper is to clarify the concept of information and discuss its mathematical models, establishing relations with physics as the most developed science.
\end{abstract}

Keywords: Information, operator, principle, theory, concept, knowledge, representation, interaction

On the one hand, information is the basic phenomenon of our world. We live in the world where information is everywhere. All knowledge is possible only because we receive, collect and produce information. People discovered existence of information and now talk of information is everywhere in our society. As Barwise and Seligman write (1997), in recent years, information became all the rage. The reason is that people are immersed in information, they cannot live without information and they are information systems themselves. The whole life is based on information processes as Loewenstein (1999) convincingly demonstrates. Information has become a key concept in sociology, political science, and the economics of the so-called information society. Thus, to better understand life, society, technology and many other things, we need to know what information is and how it behaves. Debons and Horne (1997) write, if information science is to be a science of information, then some clear understanding of the object in question requires definition.

On the other hand, the actual nature of the information and knowledge produced and distributed by information technology remains abstract and actually undefined. Even more, many researchers assume that this diversity of information uses forms an insurmountable obstacle to creation of a unified comprehensible information theory. For instance, Shannon (1993) wrote that it is hardly to be expected that a single concept of information would satisfactorily account for the numerous possible applications of this general field. Gofman (1970) and Gilligan (1994) argued that the term information has been used in so many different and sometimes incommensurable ways, forms and contexts that it is not even worthwhile to elaborate a single conceptualization achieving general agreement. Capuro, Fleissner, and Hofkirchner (1999) even gave an informal proof of the, socalled, Capuro trilemma that implies impossibility of a comprising concept of information.

In spite of this some researchers has believed in a possibility to achieve a unified definition of information by building a unified theory of information (cf., for example, Hofkirchner, 1999; 2009). The reality supported this belief and such a unified theory called the general theory of information has been created (Burgin, 2010). In it, utilization of a new type of definition, which is called a parametric definition, made it possible to adequately and comprehensively define information.

Parametric systems (parametric curves, parametric equations, parametric functions, etc.) have been frequently used in mathematics and its applications for a long time. For instance, a parametric curve in a plane is defined by two functions $f(t)$ and $g(t)$, while a parametric curve in space has the following form: $(f(t), g(t), h(t))$ where parameter $t$ takes values in some interval of real numbers. Parameters used in mathematics and science are, as a rule, only numerical and are considered as quantities that define certain characteristics of systems. For instance, in probability theory, the 
normal distribution has the mean $\mu$ and the standard deviation $\sigma$ as parameters. A functional parameter is utilized for constructing families of non-Diophantine arithmetics (Burgin, 1997).

In the case of the general theory of information, the parameter is even more general. The parametric definition of information utilizes a system parameter. Namely, an infological system plays the role of a parameter that discerns different kinds of information, e.g., social, personal, chemical, biological, genetic, or cognitive, and combines all existing kinds and types of information in one general concept "information". This parametric approach provides tool for building the general theory of information as a synthetic approach, which organizes and encompasses all main directions in information theory (Burgin, 2010).

If it is suggested that an information theory is a general, or unifying, theory of information, then it is necessary to demonstrated that this theory comprises all other directions in information theory, as well as covers all uses of the term information. Assessing the general theory of information by this condition, we see that in the book (Burgin, 2010), it is demonstrated that the general theory of information includes all major directions in information theory with core mathematical models. Actually, it is possible to this for any existing now direction in information theory. Moreover, the general theory of information either encompasses other uses of the term information or puts these uses into a correct context by assigning to them other concepts from the general theory of information. In such a way, the general theory of information covers all uses of the term information.

In the first section of the paper, a brief synopsis of the information theory history is given. In the second section, problems with correct understanding of the phenomenon information are discussed. In the third section, meta-axiomatic foundations of the general theory of information are presented. In the fourth section, operator mathematical models of information in the context of the general theory of information are described. In conclusion, some open problems in information theory are given.

\section{A Brief Synopsis of the Information Theory History}

It was natural that the beginning of information theory as a scientific discipline was shaped by mundane definitions of information and the anthropomorphic approach of researchers. Their reasoning was based on experience of generations who comprehended information as understandable messages. Analysis of messages and their exchange in communication brought forth the statistical approach to information, which materialized in Hartley-Shannon's theory (Shannon, 1948) and to some extent in Fisher's theory (Fisher, 1925). These theories tell nothing about information per se but give very efficient information measures. Due to this efficiency Hartley-Shannon's information theory became domineering in the information research, monopolizing even the name information theory because many researchers attribute this name exclusively to Hartley-Shannon's information theory. Many books with the title Information Theory contain information only about Hartley-Shannon's information theory (cf., for example, (Ash, 1965; Cover \& Thomas, 2006; Goldman, 1968) in spite of persuasive demonstration of limitations of this theory by other researchers (cf. for example Bar-Hillel \& Carnap, 1958; Brukner \& Zeilinger, 2001; Marschak, 1954) and elaboration of other approaches in information theory.

The second component of the anthropomorphic image of information instigated the development of semantic information theory (Bar-Hillel \& Carnap, 1958), which tryed to overcome limitations of the statistical approach to information. The main incentive of this approach is representation of the meaning of information by logical means.

In the process of the society development, the role of information was growing and the understanding of this role increased. Researchers started to see information processes and their impact in different areas of social life. This brought into being economical information theories (cf. Marschak, 1954) and other pragmatic information theories (cf. Harkevitch, 1960; Bongard, 1970; Hilton, 1981; Schepanski \& Uecker, 1983).

At the same time, understanding of the second component of the anthropomorphic image of information instigated the development of algorithmic information theory (Solomonoff, 1964; Kol- 
mogorov, 1965; Chaitin, 1966), as well as studies of information transformations (Mazur, 1984; Chechkin, 1991) and information flow (Dretske, 1981; Barwise \& Seligman, 1997).

All these and many other directions and ideas in information theory made possible creation of the general theory of information (Burgin, 2010).

\section{What Holds Back Understanding of Information}

Two main misconceptions in information studies are (Burgin, 2003a; 2003b): (1) comprehending the information carrier as information itself (information per se), e.g., in many sources, we can read that information is a message or information is a kind of data (Burgin, 2004b) and (2) calling a measure of information by the name information, e.g., Shannon information (cf. Brukner \& Zeilinger, 2001; Timpson, 2003) or Fisher information (cf. for example Frieden, 1998).

At first, let us analyze the differences between information, information carrier and information representation. To better grasp the situation, consider a letter in an envelope. Receiving an envelope with a letter, you do not think that this is the letter - you understand that the letter is inside the envelope. In turn, the letter you receive gives you information because this letter contains a text that carries this information. In turn, the text contains a message and the message contains information.

It is easier to understand the differences between the envelope and the letter in it than the differences between the message and information in it. However, both situations are very similar.

According to the general theory of information, information I for a system $R$ has to in an object that can interact with the system $R$. Otherwise, the system $R$ will not be able to receive this information $I$. Such an object is called the carrier of information $I$. For instance knowledge and data are carriers of information (Burgin, 2004a).

However, not every carrier of information I represents this information. For instance, the envelope that contains a letter is only a carrier of information in the letter, while the text of the letter represents this information. Another example is a box without any indication of what is inside. It is a carrier of its content. However, when something is written on the box describing its content or the box has a specific size and form, e.g., a shoe box, then the box becomes a representation of its content. Although, sometimes it is misleading: imagine a book in a shoe box. In this case, the shoe box is only a carrier but not a representation of the book in it.

At the same time, it is useful to know that for the postal service, a letter is any envelope just sent by mail even if this envelope is empty. This shows that the same word may have different meaning in different contexts. In a similar way, data may be treated as information from the point of view of information storage or a message may be called information in the context of technical communication devices.

Now let us analyze the differences between information and its measure, taking the Shannon's measure of information (Shannon, 1948), which is called entropy, is the most popular information measure and according to which information in a message $m$ is measured by the formula

$$
H(m)=H\left(p_{1}, p_{2}, \ldots, p_{n}\right)=-\sum_{i=1}^{n} p_{i} \cdot \log _{2} p_{i}
$$

We can see that according to this formula, two very different messages $A$ and $B$ can have the same entropy $H(A)=H(B)$. For instance, the message $A$ informs a person $P$ who lives in Paris that it was raining in Tokyo last week, while the message $B$ informs the same person $P$ that her son will come to her next from New York. If the probability $p$ of both events is the same, then both messages will contain the same quantity of information

$$
H(A)=H(B)=p \cdot \log _{2} p
$$

However, information in both letters is essentially different. Thus the measure of information is not information. 
It is interesting to know that information measures have been created without proper understanding of what is measured. This is similar to the general situation in science. For instance, it was possible to measure volume, mass (weight) and temperature of water before chemists determined the molecular structure of water.

\section{Meta-Axiomatic Foundations of the General Theory of Information}

The general theory of information is constructed on three levels: conceptual, methodological (also called meta-theoretical) and theoretical. On the conceptual level, the essence of information as a dynamic object playing a pivotal role in all walks of reality is explicated, clarifying a quantity of misconceptions, fallacies and illusions. Methodological (meta-theoretical) level is based on two classes of principles (ontological and axiological principles) and their relations. On the theoretical level, axioms of structures used and axioms reflecting features of information are introduced and utilized for building models of information and related phenomena, e.g., information flow or information processing.

To clarify the concept of information, we consider here the basic ontological principles. The first of them separates local and global approaches to information definition, i.e., in what context information is defined.

\subsection{Ontological Principle 01 (the Locality Principle).}

It is necessary to separate information in general from information (or a portion of information) for a system $R$.

In other words, empirically, it is possible to speak only about information (or a portion of information) for a system.

\subsection{Definition 1}

The system $R$ with respect to which some information is considered is called the receiver, receptor or recipient of this information.

Such a receiver/recipient can be a person, community, class of students, audience in a theater, animal, bird, fish, computer, network, database and so on.

The Locality Principle explicates an important property of information, but says nothing what information is. The essence of information is described by the second ontological principle, which has several forms.

\subsection{Ontological Principle 02 (the General Transformation Principle)}

In a broad sense, information for a system $R$ is a capacity to cause changes in the system $R$.

Thus, we may understand information in a broad sense as a capacity (ability or potency) of things, both material and abstract, to change other things. Information exists in the form of portions of information. Informally, a portion of information is such information that can be separated from other information. Information is, as a rule, about something. What information is about is called the object of this information. Thus, we can see that all kinds and types of information are encompassed by the Ontological Principle O2. In the most concise form, it is demonstrated in (Burgin, 2010).

However, the common usage of the word information does not imply such wide generalizations as the Ontological Principle $\mathrm{O} 2$ implies, and we need a more restricted theoretical meaning because an adequate theory, whether of information or of anything else, must be in significant accord with our common ways of thinking and talking about what the theory is about, else there is the danger that theory is not about what it purports to be about.

Information in a proper sense is defined of structural infological systems. In essence, any subsystem of a system may be considered as its infological system. However, information in a proper 
sense acts on structural infological systems. An infological system structural is structural if all its elements are structures. For example, systems of knowledge are structures.

To achieve precision in the information definition, we do two conceptual steps. At first, we make the concept of information relative to the chosen infological system $\operatorname{IF}(R)$ of the system $R$ and then we select a specific class of infological systems to specify information in the strict sense. That is why it is impossible and, as well as, counterproductive to give an exact and thus, too rigid and restricted definition of an infological system. Indeed, information is a very rich and widespread phenomenon to be reflected by a restricted rigid definition (cf. for example Capurro, Fleissner, \& Hofkirchner, 1999; Melik-Gaikazyan, 1997).

\subsection{Ontological Principle 02g (the Relativized Transformation Principle)}

Information for a system $R$ relative to the infological system $\operatorname{IF}(R)$ is a capacity to cause changes in the system $\operatorname{IF}(R)$. Elements from $\operatorname{IF}(R)$ are called infological elements.

Even more restricted is the following principle.

\subsection{Ontological Principle 02a (the Special Transformation Principle)}

Information in the strict sense or proper information or, simply, information for a system $R$, is a capacity to change structural infological elements from an infological system $\operatorname{IF}(R)$ of the system $R$.

To better understand how infological system can help to explicate the concept of information in the strict sense, we consider cognitive infological systems.

An infological system $\operatorname{IF}(R)$ of the system $R$ is called cognitive if $\mathrm{IF}(R)$ contains (stores) elements or constituents of cognition, such as knowledge, data, ideas, fantasies, abstractions, beliefs, etc. A cognitive infological system of a system $R$ is denoted by $\operatorname{CIF}(R)$ and is related to cognitive information.

\subsection{Ontological Principle 02c (the Cognitive Transformation Principle)}

Cognitive information for a system $R$, is a capacity to cause changes in the cognitive infological system $\operatorname{IFC}(R)$ of the system $R$.

So, we come to the situation where the concept of information is considered on three basic levels of generality:

- Information in a broad sense is considered when there are no restrictions on the infological system (cf. Ontological Principle O2).

- Information in the strict sense is considered when the infological system consists of structural elements (cf. Ontological Principle O2a).

- Cognitive information is considered when the infological system consists of cognitive structures, such as knowledge, beliefs, ideas, images, etc. (cf. Ontological Principle O2c).

Consequently, we have three levels of information understanding:

- Information in a broad sense for a system $R$ is a capability (potential) to change (transform) this system in any way.

- Information in the strict sense for a system $R$ is a capability (potential) to change (transform) structural components of this system, e.g., cognitive information changes knowledge of the system, affective information changes the state of the system, while effective information changes system orientation (Burgin, 2010).

- Cognitive information for a system $R$ is a capability (potential) to change (transform) the cognitive subsystem of this system. 
Let us explicate other properties of information, taking into consideration a portion I of information for a system $R$.

\subsection{Ontological Principle 03 (the Embodiment Principle)}

For any portion of information $I$, there is always a carrier $C$ of this portion of information for a system $R$. The substance $C$ that is a carrier of the portion of information $I$ is called the physical, or material, carrier of $I$.

\subsection{Ontological Principle 04 (the Representability Principle)}

For any portion of information $I$, there is always a representation $C$ of this portion of information for a system $R$. People empirically observed that for information to become available, the carrier must interact with a receptor that is capable of detecting information the carrier contained. The empirical fact is represented by the following principle.

\subsection{Ontological Principle 05 (the Interaction Principle)}

A transaction/transition/transmission of information goes on only in some interaction of $C$ with $R$. However, being necessary, interaction is not sufficient for information transmission. Thus, we need one more principle.

\subsection{Ontological Principle 06 (the Actuality Principle)}

A system $R$ accepts a portion of information I only if the transaction/transition/transmission causes corresponding transformations in $R$.

For instance, if after reading this paper, your knowledge remains the same, you do not accept cognitive information from this text. In a general case, when the recipient's knowledge structure was not changed, there is no cognitive information reception.

\subsection{Ontological Principle 07 (the Multiplicity Principle)}

One and the same carrier $C$ can contain different portions of information for one and the same system $R$.

\section{Operator Models of Information}

The mathematical stratum of the general theory of information is build as an operator theory in information spaces based on principles of this theory, which are translated into postulates and axioms. Informally, an information space is a space where information functions (acts). In the functional approach, information spaces are constructed as state or phase spaces of infological systems. It is possible to use different mathematical structures for state/phase representation. Here we are mostly interested in the functional approach when state and phase spaces are function spaces.

According to the Ontological Principle $\mathrm{O} 2$, the essence of information in a broad sense is change (transformation) in a system $R$. In a similar way, according to the Ontological Principle O2g, the essence of information in the strict sense is the change (transformation) in the infological system IF $(R)$ of $R$. So, building a mathematical model of information, it is necessary to represent the system $R$ and/or $\mathrm{IF}(R)$ by a mathematical structure and to portray changes in $R$ and/or in its infological system $\operatorname{IF}(R)$ by transformations in this structure. This brings us to the conclusion that the basic structure for the mathematical representation of information is a system space $L$, in which the system $R$, or its infological system $\operatorname{IF}(R)$, is represented. Systems that are (potential) receivers of information are represented by points in the space $L$. Note that elements (points) of the space $L$ may have a sophisticated structure. For instance, $L$ can be a space of functions, of semantic networks or of classifications. 


\subsection{Example 1}

In control engineering and the theory of automata, a state space is a mathematical model that represents a system as a set of input, output and state variables, which form a function space. State space of a system is an example of a system space $L$. Dynamics of the system in question is represented by transition rules.

For systems studied in control engineering, variables are related by differential equations that describe evolution of the system. To make this possible and to abstract from the nature of inputs, outputs and states, the variables are expressed as vectors, while the differential and algebraic equations are written in matrix form.

For abstract automata, such as finite automata and Turing machines, variables are symbolic systems and evolution of the whole system, i.e., abstract automaton, is described by transition rules or transition function.

\subsection{Example 2}

The state space, also called the phase space, of a physical system is also an example of a system space $L$. In mathematics and physics, state space is the space in which all possible states of a system are represented, with each possible state of the system corresponding to one unique point in the state space. For mechanical systems, the state space usually consists of all possible values of position and momentum variables. A state is, as a rule, a region of the state space. In thermodynamics, a phase is a region of space where matter lies in a given physical configuration, like for example of a liquid phase, or a solid phase, etc.

Quantum mechanics, in its most general formulation, is a theory of abstract operators (observables) acting on an abstract state space (usually, it is a Hilbert space), where the observables represent physically-observable quantities and the state space represents the possible states of the system under study.

In quantum field theory, the phase space is a Hilbert space $H$, i.e., an infinite dimensional complete vector space with a scalar product, while physical systems are represented by vectors in $H$. It is assumed that this vector contains all information on the considered physical system (cf. for example Bjorken \& Drell, 1965).

\subsection{Definition 1}

- A system $R$ is represented in a system space $L$ when states or phases of $R$ are corresponded to points of $L$.

- The system $R$ is statically represented in the space $L$ when states of $R$ are corresponded to points of $L$. Such a static representation makes $L$ a state space of $R$.

- The system $R$ is processually represented in the space $L$ when phases of $R$ are corresponded to points of $L$. Such a processual representation makes $L$ a phase space of $R$.

We distinguish two kinds of system representations: state and phase representations, assuming that different states and phases of systems are distinguishable. A state of a system $R$ is a static characterization of this system. A phase of a system $R$ is a process that is taking place in this system. It is possible to consider a phase of a system as a dynamic state, i.e., a state in which transition (change) in the system takes place (is going). For instance, static states of a printer are off and on. Printing and Ready are dynamic states, or phases, of a printer. Thus, a phase of a system $R$ is a dynamic characterization of this system.

A state representation is the triad $\left(\mathrm{St}(R), r_{\mathrm{st}}, L\right)$. Here $\mathrm{St}(R)$ is the set of all possible states of $R$, $r_{\text {st }}$ is a binary relation between $\operatorname{St}(R)$ and $L$, and $L$ is a set with some structure and $L$ is called the state representation space, or simply, the state space, of $R$. 
A phase representation is the triad $\left(\mathrm{Ph}(R), r_{\mathrm{ph}}, L\right)$. Here $\mathrm{Ph}(R)$ is the set of all possible phases of $R, r_{\mathrm{ph}}$ is a binary relation between $\mathrm{Ph}(R)$ and $L$, and $L$ is a set with some structure called the phase representation space, or simply, the phase space, of $R$.

\subsection{Example 3}

An important example of a representation space $L$ for a cognitive infological system is the set of all propositions in some logical language $C$. Propositions are functions that take truth values. This representation space is used in the information theory of Bar-Hillel and Carnap (1958), as well as in the multiplicity of works on cognitive and intelligent agents (cf. for example Halpern \& Moses, 1985). It is also possible to consider a propositional calculus, predicate calculus, propositional variety or predicate variety (Burgin, 2004b) as a representation space for a cognitive infological system.

\subsection{Example 4}

The category of all classifications from the model of Barwise and Seligman (1997) is an example of a state space $L$. This is also a function space as a classification is a function. In addition, such a space has the algebraic structure of a category with classifications as its objects and infomorphisms as morphisms. Transformations of such categorical spaces are endofunctors.

It is possible that the system space $L$ is a mathematical structure, e.g., a linear space, or it can be a set, universe or system of such structures. For instance, when we take the set of all classifications in the sense of Barwise and Seligman (1997) or the set of all semantic networks as $L$, it has the structure of an algebraic category. In physics, state spaces have the structure of a linear space.

\subsection{Definition 2}

The set $\mathrm{U}(R)$ of all points from $L$ that are corresponded to states (phases) of $R$ are called the state (phase) representation domain of $R$ in $L$.

The domain $U(R)$ of $L$ usually is treated as the state space of $R$ where different points correspond to different states of $R$ or as the phase space of $R$ where different points correspond to different phases of $R$. Very often we have $U(R)=L$.

Transformations of systems have different forms. A transformation can be a process, phase, state transition, multistate transition, i.e., a composition of state transitions, etc. For instance, a state transition of a system $R$ is a transition of $R$ from one state to another.

A state-transition representation is the triad $\left(\operatorname{Trans}(R), r_{\text {sttr }}, L\right)$. Here $\operatorname{Trans}(R)$ is the set of all state transitions of the system $R$ and $r_{\text {sttr }}$ is a binary relation between $\operatorname{Trans}(R)$ and the set of pairs $(a, f(a))$ with $a \in L$. Operators on states of $R$ correspond to endomorphisms of $L$, i.e., mappings of $L$ into itself that preserve a chosen structure in $L$.

\subsection{Definition 3}

- A system $R$ is dynamically represented in the space $K$ if transformations (e.g., transitions of states) of $R$ are corresponded to elements of $K$.

- A system $R$ is transitionally represented in the space $L$ if transitions of states of $R$ are corresponded to transformations of $L$.

To build a mathematical model of information, we consider a class $\mathbf{K}$ of systems, which are receptors of information, a set IT of infological system types, and a set $\mathbf{L}$ of representation spaces for infological systems of systems from $\mathbf{K}$. In addition, for each system $R$ from $\mathbf{K}$ and infological system $\mathrm{IF}(R)$ with a type from IT, a representation ( $\left.\mathrm{St}(\mathrm{IF}(R)), r_{\mathrm{st}}, L\right)$ with $L \in \mathrm{L}$ is fixed. 


\subsection{Definition 4}

- A general information operator $I$ in $L$ is a partial mapping $I: \bigcup_{L=L} L \rightarrow \bigcup_{L \mathrm{~L} L} L$.

- A (particular) information operator $I$ in $L$ is a partial mapping $I: L \rightarrow L$.

Each information operator represents a. portion of information. Note that a union of several portions of information is also a portion of information (Burgin, 2010). Here we typically consider functional information operators acting in function spaces.

We denote the set of all general information operators in $\mathbf{L}$ by $O p \mathbf{L}$, the set of all total information operators in $\mathbf{L}$ by $O p t \mathbf{L}$, the set of all information operators in $L$ by $O p L$ and the set of all total information operators in $L$ by Opt $L$.

\subsection{Example 5}

A proposition $p$ induces an information operator $A_{p}$ on systems of (logical) worlds used in semantic information theories. A logical world consists of all true statements/propositions about all entities from this world. If $\mathrm{W}$ is a system of logical worlds, then the information operator $A_{p}$ excludes all worlds where $p$ is not true, i.e., all worlds inconsistent with $p$.

At the same time, a proposition $p$ induces an information operator $A_{p}$ on states of a chosen universe $U$ according to semantic information theories. States of $U$ are consistent assignments of truth values to primitive propositions from a propositional language $L$. The information operator $A_{p}$ excludes all states where $p$ is not true, i.e., all states inconsistent with $p$.

\subsection{Example 6}

A portion of cognitive (epistemic in the sense of Mizzaro, 2001) information I induces an information operator $A_{\text {l }}$ on systems of knowledge systems. In the theory of epistemic information, it is assumed that a knowledge system $\mathbf{K}$ consists of knowledge items. It is possible to take elementary knowledge units built in (Burgin, 2004a) or elementary propositions/predicates as such knowledge items.

\subsection{Example 7}

Infomorphisms from the theory of information flow (Barwise \& Seligman, 1997) are examples of information operators. Endofunctors in the category IFlow with classifications as its objects and infomorphisms as morphisms are also examples of information operators.

\subsection{Example 8}

Ultramappings and ultraoperators from the operator information theory are examples of information operators (Chechkin, 1991). Endofunctors from the category $\operatorname{IUMap}(X)$ with the set $\operatorname{Data}(X)$ of all elementary data on all points from $X$ as the set of its objects and ultramappings as morphisms are also examples of information operators. In a similar way, endofunctors from the category IUOp $(X)$ with general iunformations as its objects and ultraoperators as morphisms give one more example of information operators.

In this model, information processes are represented by operator algebras and operator dynamical systems. These systems can be continuous or discrete. For instance, information processing in contemporary computers is represented by discrete operator dynamical systems.

Different properties of functional information operators, their transformations and categories are studied in (Burgin, 2010). 


\section{Conclusion}

Thus, we have demonstrated what misconceptions hindered elaboration of the unified concept of information, clarified this concept, allowing unification of diverse understandings and usages of the term information in the context of the general theory of information, and explicated a mathematical model of information.

In this context, it might be useful to suggest several problems for future research.

- Build an axiomatic description of information spaces and study their properties in this context.

- Define and study algebras of information operators for various information spaces.

- Develop mathematical theory of information processes.

\section{References}

Ash, R. B. (1965). Information theory. New York, NY: Interscience.

Bar-Hillel, Y. \& Carnap, R. (1958). Semantic information. British J. of Philosophical Sciences, 4(3), 147-157.

Barwise, J. \& Seligman, J. ( 1997). Information flow: The logic of distributed systems: Cambridge tracts in theoretical computer science (Vol. 44). Cambridge, UK: Cambridge University Press.

Bjorken, J. D. \& Drell, S. D. (1965). Relativistic quantum fields. Columbus, OH: McGraw-Hill Book Company.

Bongard, M. M. (1970). Pattern recognition. Rochelle Park, NJ: Hayden Book Co., Spartan Books.

Brukner, C. \& Zeilinger, A. (2001). Conceptual inadequacy of the Shannon information in quantum measurements. Physical Review A, 63, 022113.

Burgin, M. (1997). Non-diophantine arithmetics or is it possible that $2+2$ is not equal to 4 ? Kiev: Ukrainian Academy of Information Sciences.

Burgin, M. (2003a). Information theory: A multifaceted model of information. Entropy, 5(2), 146-160.

Burgin, M. (2003b). Information: Problem, paradoxes, and solutions. tripleC, 1(1), 53-70.

Burgin, M. (2004a). Data, information, and knowledge. Information, 7(1), 47-57.

Burgin, M. (2004b). Logical tools for program integration and interoperability. In Proceedings of the IASTED International Conference on Software Engineering and Applications (pp. 743-748). Cambridge, MA: MIT.

Burgin, M. (2010). Theory of information: Fundamentality, diversity and unification. New York, NY; London, UK; Singapore: World Scientific.

Capurro, R., Fleissner, P., \& Hofkirchner, W. (1999). Is a unified theory of information feasible? In W. Hofkirchner (Ed.), The quest for a unified theory of information: Proceedings of the 2nd International Conference on the Foundations of Information Science (pp. 9-30). Amsterdam: Gordon and Breach.

Chaitin, G. J. (1966). On the length of programs for computing finite binary sequences. J. Association for Computing Machinery, 13(4), 547-569.

Chechkin, A. V. (1991). Mathematical informatics. Moscow: Nauka. (in Russian)

Cover, T. M. \& Thomas, J. A. (2006). Elements of information theory. New York, NY: Wiley-Interscience.

Debons, A. \& Horne, E. E. (1997). NATO Advanced Study Institutes of Information Studies and Foundations. Journal of the American Society for Information Science and Technology, 48(9), 794-803.

Dretske, F. I. (1981). Knowledge and the flow of information. Oxford, UK: Basil Blackwell.

Fisher, R. A. (1925) Theory of statistical estimation. Proceedings of the Cambridge Philosophical Society, 22, 700-725.

Flükiger, D. F. (1999). Towards a unified concept of information: Presentation of a new approach. In W. Hofkirchner (Ed.), The quest for a unified theory of information: Proceedings of the 2nd International Conference on the Foundations of Information Science (pp. 101-111). Amsterdam: Gordon and Breach.

Frieden, R. B. (1998). Physics from Fisher information. Cambridge, UK: Cambridge University Press.

Fuchs, C. A. (2002). Quantum mechanics as quantum information. Retrieved from: http://www.perimeterinstitute.ca/personal/cfuchs/VaccineQPH.pdf.

Gilligan, J. (1994). Patterns on glass: The language games of information, Symposium on the Philosophical and Logical Aspects of Information Systems (pp. 20-22). Bristol: University of the West of England.

Gofman, W. (1970). Information science: Discipline or disappearence? Aslib Proceedings, 22, 589-585.

Goldman, S. (1968). Information theory. Mineola, NY: Dover Publications.

Halpern, J.Y. \& Moses, Y. (1985). Towards a theory of knowledge and ignorance. In K. R. Apt (Ed.), Logics and Models of Concurrent Systems (pp. 459-476) New York: Springer.

Harkevich, A. (1960). On the value of information. Problems of Cybernetics, 4, 2-10.

CC: Creative Commons License, 2011. 
Hilton, R. W. (1981). The determinants of information value: Synthesizing some general results. Management Science, 27(1), 57-64.

Hofkirchner, W. (Ed.). (1999). The quest for a unified theory of information: Proceedings of the 2nd International Conference on the Foundations of Information Science. Amsterdam, NL: Gordon and Breach.

Hofkirchner, W. (2009). How to achieve a unified theory of information. tripleC, 7(2), 357-368.

Kolmogorov, A. N. (1965). Three approaches to the definition of the quantity of information. Problems of Information Transmission, 1, 3-11.

Loewenstein, W. R. (1999). The touchstone of life: Molecular information, cell communication, and the foundation of life. Oxford, UK; New York, NY: Oxford University Press.

Marschak, J. (1954). Towards an economic theory of organization and information. In R. M. Thrall, C. H. Coombs, \& R. L. Davies (Eds.), Decision processes (pp. 187-220). New York, NY: Wiley and Sons.

Mazur, M. (1970). Jakosciowa Teoria Informacji .Warszawa, PL: PAN.

Melik-Gaikazyan, I. V. (1997). Information processes and reality. Moscow: Nauka. (in Russian, English summary)

Mizzaro, S. (2001). Towards a theory of epistemic information. Information Modelling and Knowledge Bases, 12, 1-20.

Schepanski, A. \& Uecker, W. (1983). Toward a positive theory of information evaluation. The Accounting Review, 58(2), 259-283.

Shannon, C. E. (1948). The mathematical theory of communication. Bell System Technical Journal, 27(1), 379-423; 27(3), 623-656.

Shannon, C. E. (1993). Collected Papers. (N. J. A. Sloane \& A. D. Wyner, Eds.). New York, NY: IEEE Press.

Solomonoff, R. (1964). A formal theory of inductive inference. Information and Control, 7(1), 1-22.

Timpson, C. G. (2003). The applicability of Shannon information in quantum mechanics and Zeilinger's foundational principle. Philosophy of Science, 70, 1233-1244.

\section{About the Author}

Mark Burgin

received his Ph.D. in mathematics from Moscow State University and Doctor of Science in logic and philosophy from the National Academy of Sciences of Ukraine. He was a Professor at the Institute of Education, Kiev; at International Solomon University, Kiev; at Kiev State University, Ukraine; and Head of the Assessment Laboratory in the Research Center of Science at the National Academy of Sciences of Ukraine. Now he is a Visiting Scholar at UCLA, Los Angeles, California, USA. Dr. Burgin is a member of New York Academy of Sciences and Honorary Professor of the Aerospace Academy of Ukraine. $\mathrm{He}$ is Editor-in-Chief of the journal Integration and Information and Associate Editor of the International Journal on Computers and their Applications. He was a member of organizing and program committees of more than 30 international conferences. Dr. Burgin is doing research, has publications, and taught courses in mathematics, computer science, information sciences, system theory, artificial intelligence, software engineering, logic, psychology, education, social sciences, and methodology of science. Dr. Burgin is the author or co-author of more than 500 published papers and 17 published books, including "Theory of Information" (2010), "Neoclassical Analysis: Calculus Closer to the Real World" (2008), "Superrecursive Algorithms" (2005), "On the Nature and Essence of Mathematics" (1998), "Intellectual Components of Creativity" (1998), "Fundamental Structures of Knowledge and Information" (1997), "Introduction to the Modern Exact Methodology of Science" (1994). 